\title{
Design of LED Luminaire for Parking Garage
}

\author{
Hao Cui* and Si-Hyun Park ${ }^{\dagger}$
}

\begin{abstract}
This study aims to design a zonal lumen for parking garage LED lightings of a slim appearance and $20 \mathrm{~W} / 2,000 \mathrm{~lm}$ capacity and to fabricate a lighting luminaire accordingly. The frame is of a one-dimensional bar type with a reverse V-shaped section, with LED chips arranged along both sides. To maximize the $60^{\circ}$ to $80^{\circ}$ zonal lumen, the geometric structure was designed with the apex of the reverse $\mathrm{V}$-shaped section at $40^{\circ}$ and both sides at $70^{\circ}$. As for the LED light source, focusing lenses with narrower full-width half-maximum (FWHM) in luminous intensity were used. A ray-tracing simulation method was utilized for the zonal lumen simulation of the given structure. An actual hardware of luminaire based on the simulation results was fabricated and characterized. The suggested model is meant to develop LED lightings with a proper level of zonal lumen required in parking garages.
\end{abstract}

Keywords: LED luminaires, Parking garage, Zonal lumen

\section{Introduction}

LEDs are a next-generation lighting source that is expected to replace light bulbs, fluorescent lamps, and halogen that have been widely used as lightings. In the history of lighting technology advancement, Thomas Edison first invented the incandescent electric lamp in 1879 , and about 60 years later, the fluorescent lamps were invented in 1938. In 1996, about 60 years later from then, white LEDs were developed. In early years, GaAs-based red and green LEDs were developed and their use was limited to indicator lamps only. After GaN-based blue LEDs were developed, however, the white LEDs were also soon developed, and it became possible to use them as LED lightings [1], [2].

As for the principle of LED operation, a semiconductor whose energy structure is adequate for direct transition when electrons undergo energy transition between a semiconductor energy bandgap (mostly III-IV compound semiconductors) is processed for a p-n junction; that is, a combination of a p-type semiconductor doped for free holes and an n-type semiconductor doped for free electrons. With forward current from outside, holes and electrons are recombined and photons of energy that correspond to an energy bandgap are radiated. Thus, the wavelength of light radiated from LEDs is concentrated in a specific bandwidth, which thus results in a specific color only. For LEDs to be used as lighting, the three primary colors-red, green, and blue-are combined and light is radiated simultaneously to create the color of white. Alternatively, complementary color mixing may be used: When blue LED and fluorescent

$\dagger$ Corresponding Author: Dept. of Electronic Engineering, Yeungnam University, Korea. (sihyun_park@ynu.ac.kr)

* Dept. of Electronic Engineering, Yeungnam University, Korea. (st7787@ynu.ac.kr)

Received: August 15, 2015; Accepted: February 12, 2016 yellow are combined, white color is produced as a result [3], [4]. Since the current is directly transited to photons in LEDs, the potential photoelectric efficiency of LEDs is as high as a maximum of $90 \%$ in wall-plug efficiency ( $5 \%$ for incandescent lamps and $40 \%$ for fluorescent lamps). Meanwhile, the lifetime is as long as 50,000 hours $(3,000$ hours for incandescent lamps, and 7,000 hours for fluorescent lamps). As a LED chip is as small as $1 \mathrm{~mm}^{2}$, it is possible to design lighting as small and slim. Unlike typical lightings, multi-step dimming control is possible. No mercury is used, and the response times are as high as 10 nano sec.

When LEDs are used as a light source, the following aspects need to be taken into account: Since LEDs are a light source that features a forward-directional radiation, light diffusion from it is difficult. The zonal light intensity control is necessary by means of reflection plates or others. When a LED lighting fixture is designed, changes in the quantity of light due to the heat generation from LED luminaires and instant overload need to be considered. As a result, about $80 \%$ of the maximum capacity of the LED fixture needs to be applied. In addition, the reduction of light penetration due to the cover and light output degradation due to loss of light inside the fixture must also to be considered.

For indoor and outdoor parking garage, outdoor gasstations and charging station, lights must be used for a long period of time and there is no need for high color rendering index (CRI) lighting. Thus, the advantages of LED lightings can be best utilized in this area of lighting business. For LED lightings to be used in such areas, however, it needs to be secured that there would be no shade or blind section. As for the zonal lumen condition of lighting luminaires for a parking garage, according to the Technical Requirement Table of North America Design Lights Consortium (DLC), $60^{\circ}$ to $80^{\circ}$ zonal lumen requires 
at least $30 \%$ of the total luminous flux, and $70^{\circ}$ to $80^{\circ}$ zonal lumen less than $25 \%$ of the total luminous flux [5]. A DLC certified company in the U.S. market of LED lighting for parking garages is BEACON, which has produced the ENDURA model [6]. This features the rectangular appearance, $300 \times 300 \times 140 \mathrm{~mm}^{3}$ in size, and a power consumption between 30 and $110 \mathrm{~W}$. In contrast, lighting fixtures with a slim appearance are differentiated in their applications from rectangular lighting models, and there is still no LED lighting fixture specifically designed for a parking garage. This study aims to design and fabricate LED luminaire for a parking garage with a slim-type appearance, whose zonal lumen satisfies DLC technical requirements, and then to characterize it.

\section{Theory for Zonal Lumen}

The most fundamental aspect to be considered in producing a lighting fixture for a parking garage is to satisfy specific zonal lumen conditions. For a general light source $I(\theta, \varphi)$, the zonal lumen $Z$. L. between $\theta_{i}$ and $\theta_{f}$ is as follows:

$$
Z . L .=\frac{\int_{\varphi=0}^{\varphi=2 \pi} \int_{\theta=\theta_{i}}^{\theta=\theta_{f}} I(\theta, \varphi) \sin \theta d \theta d \varphi}{\int_{\varphi=0}^{\varphi=2 \pi} \int_{\theta=0}^{\theta=\pi / 2} I(\theta, \varphi) \sin \theta d \theta d \varphi}
$$

Here, $\theta$ is the angle between the $z$ axis in the positive direction and the straight line connected to the origin, and $\varphi$ is the angle between the $x$ axis in the positive direction and the projected line onto $x-y$ plan of the straight line connected to the origin. When the light source is symmetrical in the direction of $\theta$, the zonal lumen to the light source $I(\theta)$ is as follows:

$$
\text { Z.L. }=\frac{\int_{\theta=\theta_{i}}^{\theta=\theta_{f}} I(\theta) \sin \theta d \theta}{\int_{\theta=0}^{\theta=\pi / 2} I(\theta) \sin \theta d \theta}
$$

In general, a single LED light source is of the following Lambertian distribution: $I(\theta)=I_{\mathrm{o}} \cos \theta$. Hence, the zonal lumen is:

$$
\text { Z.L. }=\cos ^{2} \theta_{i}-\cos ^{2} \theta_{f}
$$

Fig. 1 shows the result of computation simulation for the light intensity distribution and zonal lumen distribution with a single LED light source itself. As shown in the diagram of the far-filed light intensity distribution in (a), the maximum luminous intensity is shown in the condition of $\theta=0^{\circ}$, and the full-width half-maximum (FWHM) in the luminous intensity distribution is $120^{\circ}$. As shown in (b),

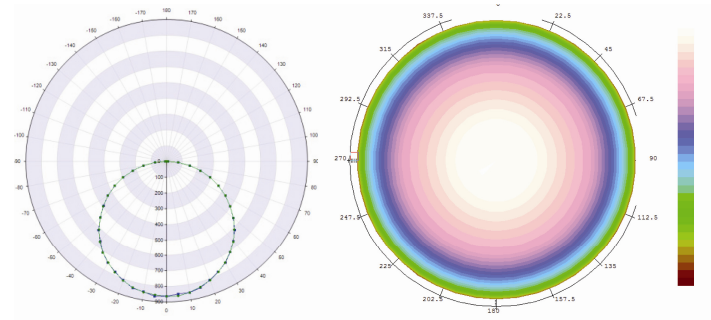

(a)

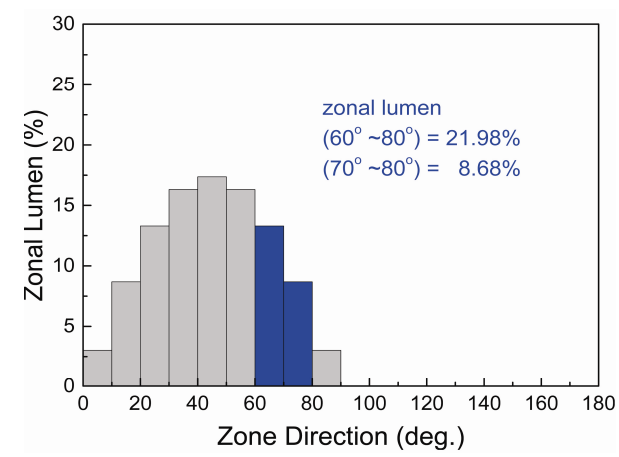

(b)

Fig. 1. Computation simulation for light intensity distribution with a single LED light source: (a) polar and raster charts; (b) zonal lumen diagram

the maximum zonal lumen is in the range of $\theta=45^{\circ}$. The zonal lumen in the area of $60^{\circ}$ to $80^{\circ}$ is $21.98 \%$ of the total luminous flux, and that in the area of $70^{\circ}$ to $80^{\circ}$ is $8.68 \%$. Thus, a single LED light source with the characteristics above requires a properly designed luminaire to secure the desired level of zonal lumen.

\section{Simulation for LED Luminaire}

A lighting luminaire for parking garages needs to meet the zonal lumen condition of DLC technical requirements. In addition, we want that the frame is designed in consideration of the slim-type appearance. As shown in Fig. 2.(a), a frame of a one-dimensional bar type with the reverse $\mathrm{V}$ shaped section is proposed. To increase the zonal lumen in the area of $60^{\circ}$ to $80^{\circ}$, the angle of the frame apex is $40^{\circ}$, and the side is $70^{\circ}$. LED chips are arranged along both side surfaces of the reverse $\mathrm{V}$ shaped frame. For the LED light source, focusing lenses are used onto the front surface of LED chip to reduce the FWHM of the luminous intensity distribution of the LED source itself. As for the optical characteristics of the frame surface, the Lambertian surface characterization is applied depending on the optical feature of the white coating panel, which is commonly used for lighting frames. LightTools program (Synopsys, Inc.) is known to be one of the most popular simulation tools for the analysis of optical phenomena in LEDs. This program is based on Monte Carlo ray-tracing method and it can be used to analyze various optical phenomena of LED 
luminaires and LED chips, including ray scattering, absorption, transmission, luminous intensity, illuminance and luminance, etc $[7,8]$. We conducted a computational simulation for the lighting fixtures, first by designing their geometrical structures using LightTools. Then over 0.1 billion rays from the source LED chips were generated in each structure for the ray-tracing simulation with numerical errors as minimized as possible. A far-field receiver was

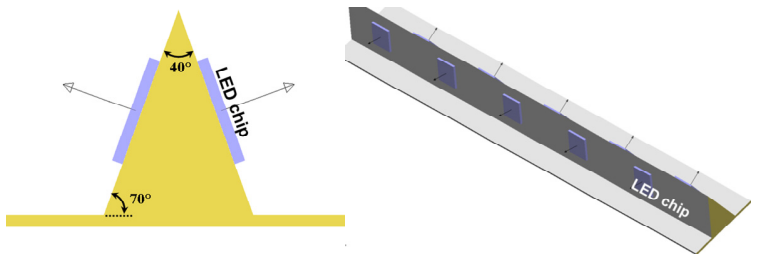

(a)

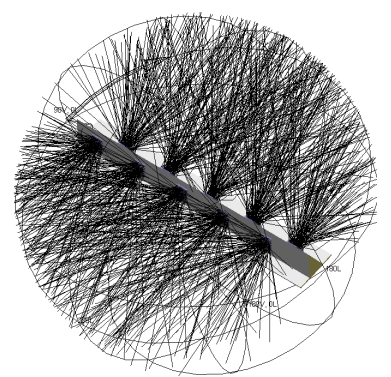

(b)

Fig. 2. (a) A bar type frame of a slim LED luminaire designed for parking garages; (b) A ray-tracing simulation for the designed lighting luminaire.

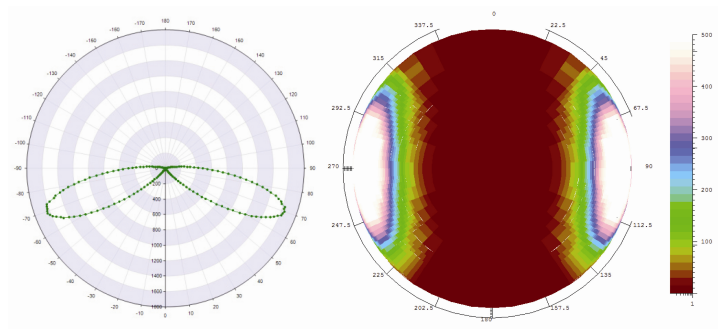

(a)

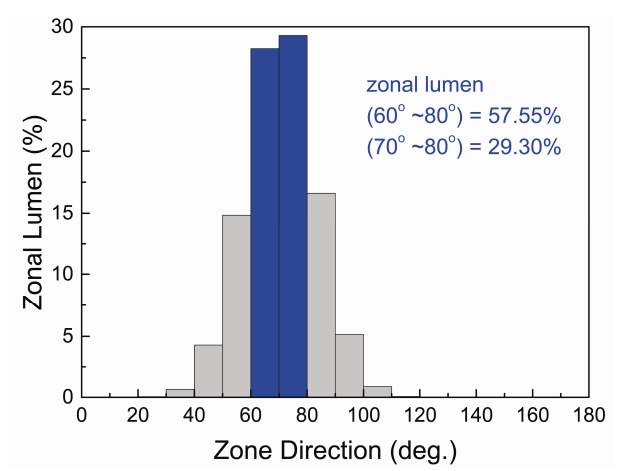

(b)

Fig. 3. The light intensity simulation for the luminaire with the focusing LED light source of FWHM $30^{\circ}$ : (a) polar and raster charts; (b) zonal lumen diagram. considered to detect the resultant rays from the whole lighting fixtures, extracting the information of light intensity distribution and zonal lumen (Fig. 2.(b)).

First of all, focusing lenses were used for the LED light source to reduce the light intensity FWHM down to $30^{\circ}$. This light source was installed in a frame of a lighting fixture whose section was of a reversed- $V$ shape as shown in Fig. 2. Fig. 3 shows the result of the simulation. As shown in (a), the maximum optical intensity appeared in the condition of $\theta=70^{\circ}$, which is because the section of that luminaire frame was of a reversed- $V$ shape with the angle of both sides at $70^{\circ}$. As shown in (b), the maximum zonal lumen was shown around $\theta=70^{\circ}$, and the zonal lumen in the area of $60^{\circ}$ to $80^{\circ}$ was $57.55 \%$ of the total luminous flux, which satisfies the desired zonal lumen condition of higher than $30 \%$. As a result of the focusing beam of the LED light source, it was possible to obtain the high value of zonal lumen in the area of maximum zonal lumen. In the area of $70^{\circ}$ to $80^{\circ}$, however, the zonal lumen was $29.30 \%$ of the total luminous flux, which was too high to satisfy the desired zonal lumen condition of lower than $25 \%$.

More alleviated focusing lenses were then used to reduce the light intensity of FWHM down to $60^{\circ}$. Fig. 4 shows the result of the light luminaire simulation. The maximum optical intensity and zonal lumen were shown in the condition of $\theta=70^{\circ}$. The zonal lumen in the area of $60^{\circ}$ to $80^{\circ}$ was $32.92 \%$ of the total luminous flux while that in the area of $70^{\circ}$ to $80^{\circ}$ was $16.62 \%$. Unlike the LED light source excessively focused at FWHM $30^{\circ}$, the LED light
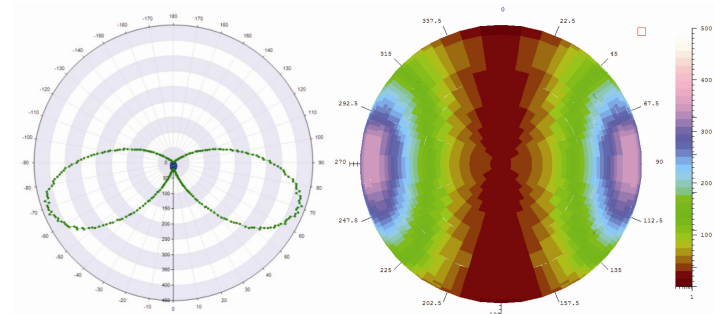

(a)

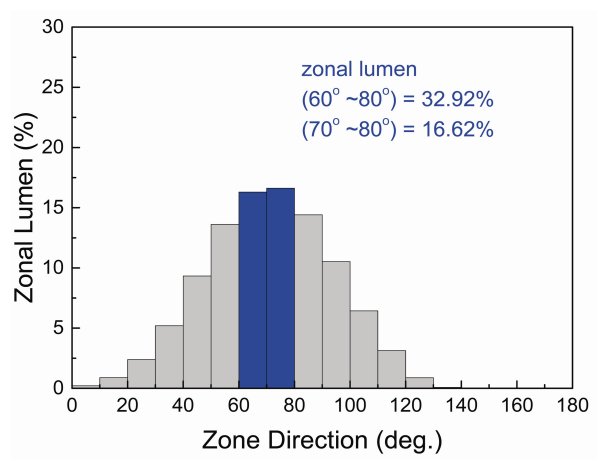

(b)

Fig. 4. The light intensity simulation for the luminaire with the focusing LED light source of FWHM $60^{\circ}$ : (a) polar and raster charts; (b) zonal lumen diagram. 

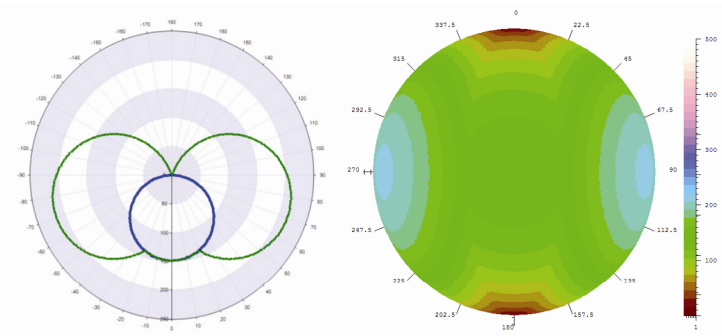

(a)

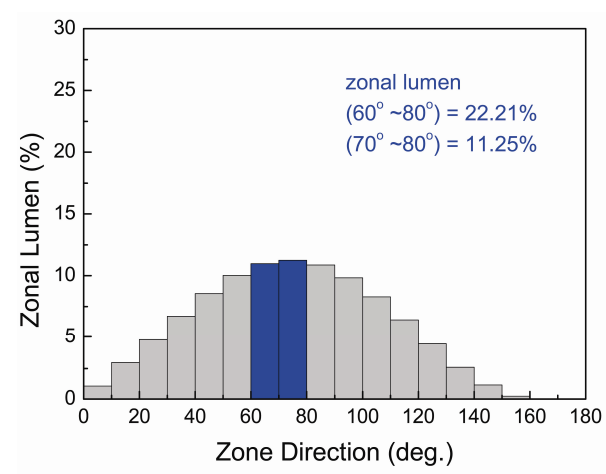

(b)

Fig. 5. The light intensity simulation for the luminaire with the non-focusing common LED light source (FWHM $120^{\circ}$ ): (a) polar and raster charts; (b) zonal lumen diagram.

source properly focused at FWHM $60^{\circ}$ showed a high level of zonal lumen in the target section while maintaining a low level of zonal lumen in other sections. This demonstrates that the desired zonal lumen condition for parking garage lighting luminaires was satisfied well.

Lastly, the LED light source whose optical intensity FWHM was $120^{\circ}$ without focusing lenses was installed in the frame structure whose section was of a reversed-V type as shown in Fig. 2, and a light intensity distribution simulation was conducted. As shown in Fig. 5, the optical intensity had double maximum points; $\theta=70^{\circ}$ was the maximum over the whole area while $\theta=0^{\circ}$ was the over a maximum local area. As for zonal lumen, the maximum zonal lumen was shown to be around $\theta=70^{\circ}$ while the zonal lumen in the area of $60^{\circ}$ to $80^{\circ}$ was $22.21 \%$ of the total luminous flux and that in the area of $70^{\circ}$ to $80^{\circ}$ was $11.25 \%$. In other words, when a common LED light source was used with no focusing lenses, the reversed-V shaped frame failed meeting the zonal lumen condition for parking garage lighting luminaires.

\section{Fabrication and Measurements of LED Luminaires}

Based on the simulation result, an LED lighting luminaire for parking garages was fabricated. In consideration of the slim appearance, the basic specifications were as follows:
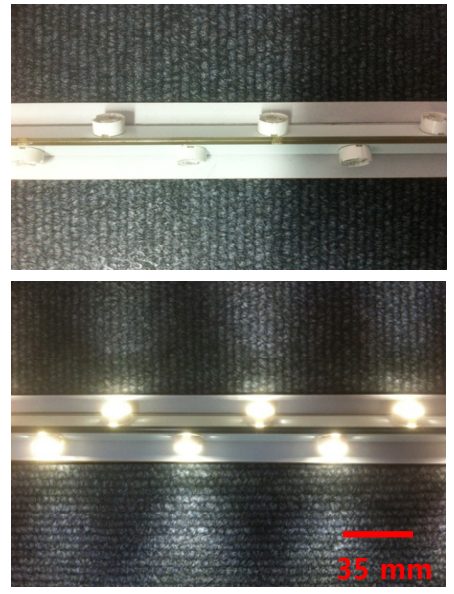

Fig. 6. The photography of the LED lighting luminaire for parking garages.
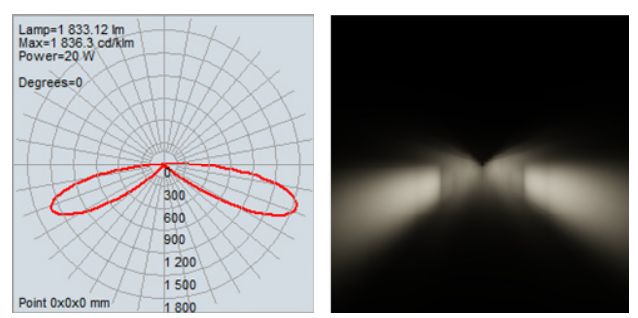

(a)

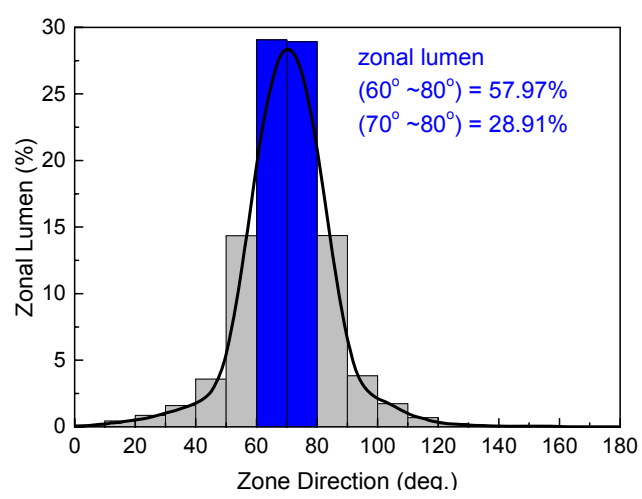

(b)

Fig. 7. The result of a light intensity measurement for the luminaire with the focusing LED light source of FWHM $30^{\circ}$. (a) polar chart of far-filed luminous intensity and corresponding luminous intensity distribution, (b) zonal lumen diagram.

$1000 \mathrm{~mm}(\mathrm{~L}) \times 50 \mathrm{~mm}(\mathrm{~W}) \times 40 \mathrm{~mm}(\mathrm{H})$ in size; $20 \mathrm{~W}$ power consumption; and 2,200 lm luminaire output. Fig. 6 shows the fabricated lighting luminaire. As shown in Fig. 2(a), the bar-type frame's sectional angles were $40^{\circ}$ and $70^{\circ}$ respectively; LEDs were arranged alternatively at both side surfaces of the reverse $\mathrm{V}$ shaped frame; and the total number of LEDs was 28 with 14 at each side. Collimated LEDs of Osram LW-W51M were adopted. For light intensity measurement, a goniophotometer (NeoLight 


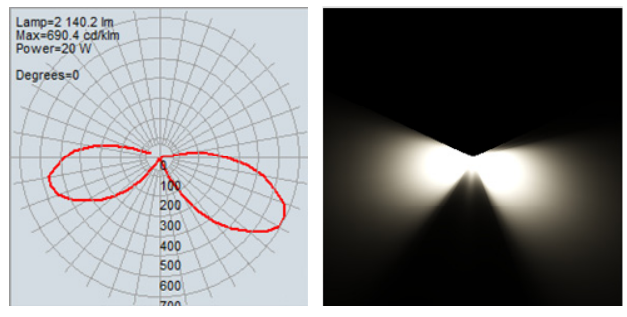

(a)

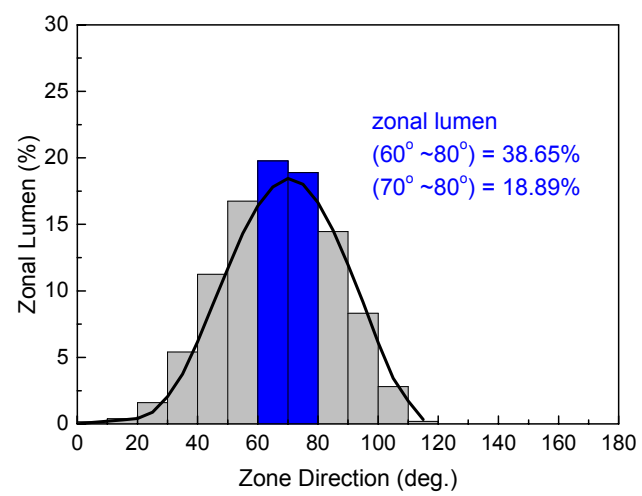

(b)

Fig. 8. The result of the light intensity measurement for the luminaire with the focusing LED light source of FWHM $60^{\circ}$ : (a) polar chart of far-filed luminous intensity and corresponding luminous intensity distribution; (b) zonal lumen diagram.

8000Series, PIMACS Co.,Ltd.) was used to measure the quantity of light at different angles, with $10 \mathrm{~m}$ distance from the light source.

For the LED light source, focusing lenses were used to reduce the FWHM of light intensity by as much as $30^{\circ}$. In this case, the light intensity distribution and zonal lumen of the lighting luminaire are shown in Fig. 7. As expected about the simulation result, the maximum light intensity was shown in the condition of $\theta=70^{\circ}$ and the maximum zonal lumen was in the condition of $\theta=70^{\circ}$. In the area of $60^{\circ}$ to $80^{\circ}$, the zonal lumen was $57.97 \%$ of the total luminous flux, and in the area of $70^{\circ}$ to $80^{\circ}, 28.91 \%$ of the total luminous flux.

For the LED light source, focusing lenses were used to reduce the FWHM of light intensity by as much as $60^{\circ}$. The light intensity distribution and zonal lumen measurements are presented in Fig. 8. The zonal lumen in the area of $60^{\circ}$ to $80^{\circ}$ was $38.65 \%$ of the total luminous flux while in the area of $70^{\circ}$ to $80^{\circ}$, it was $18.89 \%$ of the total luminous flux, which indicates that as expected about the simulation result, the requirements of zonal lumen for parking garage lighting luminaires were satisfied well.

\section{Conclusion}

This study applies two elements — a luminaire frame structure whose section is of a reversed-V shape and a properly focused LED light source — to design and fabricate a slim light luminaire which satisfies DLC technical requirements for parking garages. The zonal lumen in the area of $60^{\circ}$ to $80^{\circ}$ should be over $30 \%$ of the total luminous flux, and that in the area of $70^{\circ}$ to $80^{\circ}$ should be under $25 \%$ of the total luminous flux. The angle of the frame apex of the reversed $\mathrm{V}$ shaped section was $40^{\circ}$, and the angle of both sides was $70^{\circ}$. LED chips were arranged along both side surfaces of the frame of a onedimensional bar shape drooping downward, which increased the zonal lumen in the area of $60^{\circ}$ to $80^{\circ}$. With the LED light source whose FWHM in luminous intensity distribution was reduced, the zonal lumen of a specific area in the whole luminaire was increased. A light intensity simulation was conducted in the ray-tracing simulation, and the luminous intensity distribution and zonal lumen were calculated for light luminaires with LED light sources in each condition of focusing FWHM. As a result, when the FWHM of the LED light source was $30^{\circ}$, the value was too large in the area of $70^{\circ}$ to $80^{\circ}$, which failed meeting the requirements. When the FWHM of the LED light source was $120^{\circ}$, the value in the area of $60^{\circ}$ to $80^{\circ}$ was too small, which also failed meeting the requirements. In contrast, when the FWHM of the focusing LED light source was $60^{\circ}$, the requirements were satisfied both in the area of $60^{\circ}$ to $80^{\circ}$ and in the area of $70^{\circ}$ to $80^{\circ}$. The designed frame was installed for the lighting luminaire in utilization of the focusing LED light source whose FWHM was set to $60^{\circ}$ based on the simulation result, and then the light intensity distribution was measured. In the area of $60^{\circ}$ to $80^{\circ}$, the value was $39 \%$ while in the area of $70^{\circ}$ to $80^{\circ}$, it was $19 \%$, both of which satisfied the requirements for the zonal lumen of parking garage luminaires. The result above can be applied to LED lighting development and the production for indoor/outdoor parking garages, outdoor gas stations, and charging stations. In addition, the result of this study may be referred to for the development of any LED lighting fixtures that are to satisfy specific zonal lumen conditions.

\section{Acknowledgements}

This work was supported by the 2014 Yeungnam University Research Grant.

\section{References}

[1] Shuji Nakamura, Masayuki Senoh and Takashi Mukai, "P-GaN/N-InGaN/N-GaN Double-Heterostructure Blue-Light-Emitting Diodes," Jpn. J. Appl. Phys., Vol. 32, No. 1A/B, pp. L8-L11, Jan. 1993.

[2] Shuji Nakamura and Michael R. Krames, 'History of Gallium-Nitride-Based Light-Emitting Diodes for 
Illumination," Proc. IEEE, Vol. 101, No. 10, pp. 2211-2220, Oct. 2013.

[3] E. F. Schubert, Light-Emitting Diode, 2nd edn, University Press. pp. 113-126, pp. 145-162, 2006,

[4] V. K. Khanna, Fundamentals of Solid-State Lighting: LEDs, OLEDs, and Their Applications in Illumination and Displays, 1st edn, CRC press. pp. 261-276, 2014,

[5] The Design Lights Consortium https://www.designlights.org/content/qp1/productsub mit/categoryspecifications

[6] Beacon Products http://www.beaconproducts.com/products/endura

[7] Kyung-Tae Kim, Kyoungwoo Jo, Jung-Ha Hwang and Ho-Ki Kwon, Si-Hyun Park, "Light Outputs of LED with Various Refractive Indices and Geometrical Structures of Encapsulants," J. Korean Phys. Soc., Vol. 57, No. 6, pp. 1779 -1783, Dec.2010.

[8] Hao Cui and Si-Hyun Park, "Numerical Simulations of the Light-Extraction Efficiency of LEDs on Sapphire Substrates Patterned with Various Polygonal Pyramids," J. Opt. Soc. Korea, Vol. 18, No. 6, pp. 772-776, Dec. 2014.

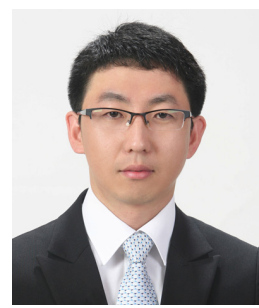

Hao Cui He received M.S degree in photonic engineering from Chosun University, Korea in 2008. He is currently working toward the Ph.D. degree in the Yeungnam University, Korea. His research interests are optical design and simulation for LED devices.

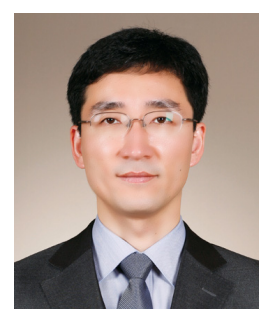

Si-Hyun Park He received Ph.D. degree specialized in optoelectronics from Seoul National University in 2004. He is currently working as Associate Professor in Department of Electronic Engineering, Yeungnam University, Korea. His current research interests are design, fabrication, characterization of optical active and passive devices such as LED and solar cell. 